\title{
Review of: "Belayer: Modeling discrete and continuous spatial variation in gene expression from spatially resolved transcriptomics"
}

\author{
Marco Antonio Mendoza-Parra ${ }^{1}$ \\ 1 Genoscope - Centre National de Séquençage
}

Potential competing interests: The author(s) declared that no potential competing interests exist.

Cong Ma et al; propose a new strategy for the analysis of spatially-resolved transcriptomes based on piecewise continous function of a single spatial variable, allowing for discontinuities in gene expression levels within the acquired spatial readouts.

The proposed strategy is inspired on a natural characteristic of tissues, namely their organization in layers (skin composed by three layers, cerebral cortex by six, etc), which might be present within the spatial transcriptome readouts.

Globally speaking the strategy is sound and the authors provided convicing results both, in simulated and in real data. Perhaps my major remark might be the fact that the application of Belayer on a variety of real data is missing. Notably its capacity to perform on high resolution data like those generated by the methodology Slide-seq; or for instance in cases in which the tissue of interest could provide a large complexity. In this context I could suggest to challenge Belayer on the analysis of the whole mouse embryo data generated with the technology DBiT-seq (Yang Liu et al; Cell 2020), which has been also recently analysed with our own tool MULTILAYER ( Moehlin et al; Cell Systems 2021). In fact, the use of MULTILAYER allowed to partition the studied tissue (whole mouse embryo) on 8 distinct "spatial communities", defined by gene co-expression signatures. Hence, it would be interesting to see the performance of Belayer for the partitioning of such complex tissue.

Finally, a related question to this type of analysis is the computing requirements for using Belayer, notably in the context of large and/or complex spatially-resolved transcriptome maps. 